\title{
Variations
}

Variations

Revue internationale de théorie critique

$20 \mid 2017$

Expériences oppositionnelles

\section{Panorama des mouvements sociaux : le Portugal, XIXe, XXe siècles.}

João Freire

\section{(2) OpenEdition}

Journals

Édition électronique

URL : http://journals.openedition.org/variations/838

DOI : 10.4000/variations.838

ISSN : 1968-3960

Éditeur

Les amis de Variations

Référence électronique

João Freire, « Panorama des mouvements sociaux : le Portugal, XIXe, XXe siècles. », Variations [En ligne], 20 | 2017, mis en ligne le 25 avril 2017, consulté le 25 février 2021. URL : http://

journals.openedition.org/variations/838; DOI : https://doi.org/10.4000/variations.838

Ce document a été généré automatiquement le 25 février 2021.

Les ami•e•s de Variations 


\title{
Panorama des mouvements sociaux : le Portugal, XIXe, XXe siècles.
}

\author{
João Freire
}

\section{Avant-propos de l'auteur}

1 Sociologue senior, professeur émérite à ISCTE-Institut Universitaire de Lisbonne. Fils de militaire, tout jeune a été officier de marine, puis déserteur pendant la guerre coloniale. Après des décennies de travaux académiques sur le travail, les technologies, les organisations et les professions, se découvre un nouveau filon de recherche dans la colonisation portugaise d'Afrique et ses anciens camarades d'armes. Le travail d'archive au prime abord ; mais aussi les exercices de mémoire et une sorte d'observation participante sont amorcés.

2 Voici un fragment jamais publié à propos de «La gaie fratrie des clercs-laïques navals (ou, paraphrasant je ne sais qui : Instead of a Spy: By a man too coward to be one), par João Freire, initié-à-moitié, profane entier et sociologue accompli».

Cela devait commencer comme suit : « Les officiers de la marine de guerre représentent un pivot institutionnel et, comme on l'a sérieusement défendu, constituent aussi une corporation, hiérarchique et nationale, fondée sur l'amalgame originale d'une identité professionnelle maritime avec une autre identité militaire ${ }^{1}$. Cependant, mis à part les termes et les détails, ils peuvent être aussi vus comme une confrérie d'initiés, de laïques qui se comportent comme de bons clercs, si l'on considère le cas portugais et, au moins, les générations qui ont connu le régime de Salazar et qui ont contribué, d'une manière ou d'une autre, à lui mettre fin et à inaugurer le cadre politique que le pays a connu depuis.

4 L'humour et la gaité sont des traits saillants d'une ambiance interpersonnelle heureuse. Le premier exige généralement l'intelligence ; la seconde peut être frivole mais peut aussi exprimer un état partagé de petits bonheurs. Quand les deux s'allient, on sera probablement face à une joyeuse communauté d'égaux, comme pouvaient l'être les cercles d'étudiants des vieilles universités traditionnelles. » 


\section{Commençons, donc} civile, laissant la besogne aux soins des juristes et autres organisateurs de l'Etat, ainsi qu'aux militaires et quand les choses se gâtent, on en vient à la guerre civile. Ici, on passe en revue le cas du Portugal dans la modernité (siècles XIX-XX'). Nous prenons le concept de mouvement social, moins par le biais interprétatif de Touraine ou Wieviorka que par une approche empirique à partir de l'existence, plus ou moins permanente et caractérisée, d'une action collective menée par des individus d'une même classe sociale comme les ouvriers ou les paysans - ou d'une même catégorie sociale (comme les jeunes ou les étudiants).

6 Le Portugal est un petit pays mais néanmoins une des nations d'Europe les plus anciennes, avec stabilité des frontières et continuité de l'Etat, une seule langue et une religion dominante. Sa position géographique y a eu sa part de responsabilité, la menace des voisins espagnols aussi ; les navigations et les découvertes du globe au XV$\mathrm{XVI}^{\mathrm{e}}$, les constructions des empires d'outre-mer ont été ses quasi-merveilleuses conséquences. Mais le pays est entré dans la modernité - industrielle, économique, politique, civile, scientifique, culturelle - en retard par rapport aux grandes nations de l'Occident. Aux temps de la Grande Révolution il était devenu un satellite politique de l'Angleterre. Avec le pays appauvri et dévasté par les armées napoléoniennes, la chute de l'Ancien Régime est arrivé en 1820 et a eu comme directe conséquence l'indépendance du Brésil, proclamée par le prince Pedro, qui est venu ensuite à Lisbonne briguer la succession de son père et, au bout d'une guerre civile (1828-1834), a réellement implanté ici un régime monarchique constitutionnel et libéral (à la manière contemporaine d'Orléans).

7 Il est peut-être utile de placer dès maintenant les principaux jalons politiques qui ont marqué la vie publique du pays, depuis cette époque jusqu'à maintenant, pour mieux cadrer notre sujet sur les mouvements sociaux et des institutions d'Etat censées assurer la paix civile. Jusqu'au milieu du XIX ${ }^{e}$, le nouveau régime a eu quelques difficultés à s'institutionnaliser, et a dû subir encore le fléau d'une jacquerie populaire-paysanne (1846-47) terminée avec intervention militaire étrangère. Mais, depuis lors, le constitutionnalisme fonctionne régulièrement : le roi, avec son pouvoir modérateur; le parlement (avec deux chambres : des députés et des pairs); le gouvernement (et ses ministères centralisés) ; la justice (avec trois instances et un tribunal suprême, et qui a aboli la peine de mort pour les crimes politiques dès 1852 et civils en 1867). L'Église est en partie intégrée à l'État comme religion officielle, s'occupant aussi de l'état civil et avec une présence importante dans la protection sociale, l'éducation, mais jouissant elle aussi de ses propres instances judiciaires. L'Armée joue un rôle politique significatif, au contraire de la Marine nationale (qui trouve un nouveau souffle avec la colonisation moderne de la fin du XIX ${ }^{\circ}$ siècle), mais elle aussi avec son propre système de justice. Enfin, ce qui reste de l'empire d'outre-mer réussi à être préservé (surtout en Afrique), donnant au gouvernement portugais une place non négligeable dans l'ordre international d'alors, notamment lors de la conférence de Berlin (1885) qui a découpé le continent noir dans ses futurs pays, selon les convenances des colonisateurs. Mais ces territoires, en dépit de leurs richesses potentielles (surtout l'Angola et le Mozambique), étaient assez délaissés et les portugais ont eu du mal à suivre l'effort de la Royal Navy 
dans l'interdiction du trafique maritime d'esclaves de l'Afrique vers le Brésil, Cuba, les États-Unis et le golfe Persique. Seules les petites îles de São Tomé et du Prince avaient créé une économie de plantation efficace (cacao, surtout), mais au prix d'une importation massive de main-d'œuvre noire (du Cap Vert, de l'Angola, etc.) suspectée de "travail forcé ». Le restant de l'empire coûtait plus cher à l'État qu'il n'en rapportait, surtout quand il s'agissait d'y envoyer des expéditions militaires pour mater les résistances indigènes.

En effet, l'endettement colonial a été une des causes qui a provoqué la révolution républicaine de 1910, tout comme la crise (politique, sociale, budgétaire) dérivée de la participation du Portugal dans la guerre de 1914-18, ce qui a amené le coup d'État militaire de 1926, et a ouvert la porte à la dictature et à l'État corporatiste de Salazar. Et c'est encore la dernière guerre coloniale en Guinée-Bissau, l'Angola et le Mozambique qui a fini par faire effondrer ce régime, lequel avait gâché les opportunités de son temps, malgré l'habileté politique du dictateur qui avait réussi a aider la victoire de Franco en Espagne, être neutre durant la deuxième guerre mondiale, être membre dès le départ de l'OTAN (et allié privilégié des États-Unis, à cause des Azores), également de la AELC/EFTA (à côté de l'Angleterre), puis, enfin, obtenir de pays comme la France, l'Allemagne, l'Italie, l'Espagne et Israël les armements et fournitures nécessaires pour mener cette guerre contre les nationalistes africains. Voici donc, comment «l'empire » a toujours été étroitement associé aux grands changements du régime politique : la perte du Brésil et l'arrivée de la monarchie libérale ; les coûts de l'occupation africaine et l'établissement de la république parlementaire ; l'épuisement des ressources dû à la Grande Guerre (en Picardie mais aussi contre les Allemands du Tanganika et du SudOuest Africain qui côtoyaient les colonies portugaises), c'était le tombeau de la dictature. Puis, finalement, encore l'Afrique avec la persistance de la lutte anticoloniale et l'établissement de la démocratie à Lisbonne.

\section{XIXe siècle}

Encore quelques mots sur la population portugaise et son économie, avant de parler des mouvements sociaux. Au milieu du XIXe siècle, la population européenne (y compris les archipels de Madère et des Azores) dépassait les 3 millions et demi, et montait à près de 6 millions en 1910. Au cours du siècle passé, elle a atteint les 8 millions et demi en 1950 et elle compte aujourd'hui quelques 10 millions d'habitants. L'urbanisation a été lente, avec deux seules grandes villes, Lisbonne et Porto, qui constituent maintenant de bonnes et grandes agglomérations métropolitaines. La population paysanne dominait largement pendant la plupart de cette période de deux cents ans : autour de $60 \%$ des actifs dans l'agriculture en 1910 , encore $50 \%$ vers 1950 ; mais cela tombe ensuite à $26 \%$ en 1970 et à $10 \%$ à la fin du siècle. Les travailleurs du secteur secondaire atteignent leur sommet quantitatif seulement vers 1980 avec $40 \%$ du total de la population active, au même niveau du tertiaire, et ces proportions commencent à fléchir depuis, selon le processus bien connu de la désindustrialisation des pays les plus développés, au profit des services.

De fait, l'industrie mécanisée n'est arrivée au pays que vers la moitié du XIX'e, en même temps que les premiers échos des luttes ouvrières en Europe et les ébauches d'un associationnisme des travailleurs salariés de nature mutualiste. Mais, dès cette époque, les élites ouvrières sont à l'écoute et répondent aux appels à la lutte de classes et aux 
tentatives d'organisation autonomes : l'Internationale arrive en 1871, avec les émotions de la Commune de Paris. Un premier parti socialiste est créé en 1878, des groupes anarchistes en 1887 ; les syndicats sont reconnus par une loi de 1891, et sous l'influence de la Charte d'Amiens du syndicalisme révolutionnaire, l'organisation syndicale se développe très rapidement à partir de 1909, la CGT étant fondée dix ans plus tard, nettement libertaire. Avec l'essor du bolchevisme, comme presque partout en Occident, une âpre concurrence s'établit dans le mouvement ouvrier mais le parti communiste créé en 1921 est dans les années 40 devenu majoritaire et, par la suite, c'est un des plus fidèles suiveurs de la politique dictée à Moscou. Vainqueur à terme de la lutte antifasciste, il fait encore aujourd'hui autour de $10 \%$ aux élections et domine la plupart des syndicats et des mairies de la banlieue de Lisbonne et de la région d'Alentejo.

11 Possédant une bonne main-d'œuvre artisanale et manufacturière, surtout dans le travail du bois, de la céramique, du verre, du textile, des produits agricoles (le vin, surtout) et de la pêche, le Portugal a raté les temps de l'industrie lourde, des applications techniques modernes, de l'électricité ou de la chimie. L'équipement industriel installé, les véhicules mécanisés (chemins de fer, navires, automobiles) étaient d'origine étrangère. La science a piétiné (malgré les bonnes connaissances accumulées en botanique et zoologie) et l'éducation a pris beaucoup de retard. L'Église catholique était profondément enracinée dans les croyances paysannes et leurs pratiques sociales. Mais sa proximité avec le pouvoir politique et les couches les plus nanties de la population a facilité la génération d'un anticléricalisme virulent vers la fin $\mathrm{du} \mathrm{XIX}^{\mathrm{e}}$ siècle, principalement dans les milieux urbains, dont le Grand Orient Lusitaniant Uni (copie conforme de France) et plusieurs organisations secrètes de «carbonaros » ont été les expressions les plus importantes.

Dans la dernière décennie de ce siècle-là, le parti républicain a pris un essor considérable, menant une critique féroce à la fois contre la monarchie et contre l'Église. Ces idées ont pénétré les classes moyennes et la petite bourgeoisie urbaine, certains secteurs populaires et beaucoup de fonctionnaires d'État, y compris les militaires. Les matelots de la flotte, qui étaient restés la plupart du temps à l'écart des débats politiques, les ont rejoints à partir de 1906, quand une mutinerie (semblable à celle du Potemkim à Odessa l'année antécédente) a éclaté à Lisbonne en pleine semaine sainte de Pâques. Les motifs de la révolte étaient d'ordre disciplinaire mais la répression qui s'en est suivi s'est chargée d'ouvrir la porte à la pénétration de l'idéologie républicaine révolutionnaire chez les marins. L'assassinat du roi et du prince héritier en 1908 a été l'œuvre d'autres radicaux mais la Marine a eu le rôle principal dans la chute violente de la monarchie et l'avènement de la république en 1910. Pendant la courte expérience de ce nouveau régime d'État les marins ont été aux postes les plus avancés de défense contre les diverses tentatives de restauration monarchique, aussi bien que contre les mouvements anti-libéraux préconisés dans les années 1920 par des secteurs conservateurs de l'armée et les admirateurs du fascisme italien. En 1936, en rapport avec la guerre civile qui couvait déjà en Espagne, a eu lieu dans le Tage la dernière manifestation de politisation des matelots, cette fois-ci sous la coupe du parti communiste, mais la prise de trois des principaux bâtiments de l'escadre n'a duré que quelques heures et les hommes déclarés coupables sont allés grossir les premiers contingents de prisonniers politiques qui arrivèrent au camp de concentration du Tarrafal (aux îles du Cap Vert) où plusieurs d'entre eux ont trouvé la mort. Cet épisode (et beaucoup d'autres tentatives armées, y compris un attentat des anarchistes contre Salazar lui-même en 1937, raté de peu), illustre la manière forte, par laquelle «l'État 
Nouveau » (militariste, corporatiste et clérical, un peu à la manière de Pétain) a traité ses opposants pendant des décennies.

Avec la propriété foncière rurale très morcelée dans le centre-nord, l'excès de bras de travail a déclenché une fuite vers l'émigration, surtout au Brésil, à partir de 1850. Au contraire, au sud du Tage prédominait absolument la grande exploitation (des latifundia, comme en Andalousie), ce qui a déterminé la consolidation d'un prolétariat rural qui, à l'époque de la république parlementaire, a su s'articuler dans les luttes pour le salaire et la dignité avec les ouvriers d'usine de la région proche de la capitale et des principaux noyaux industriels dispersés dans le territoire: Porto, Coimbra, Covilhã, Marinha Grande, Tomar, Setubal et les ports de pêche de l'Algarve. Cependant, l'émigration est devenue un phénomène démographique structurel du Portugal moderne, excédant largement la capacité de l'industrie et des services créés en ville à absorber les flux de l'exode rural. Au XXe en plus du Brésil, la métropole a réussi à canaliser une partie de ces mouvements vers les colonies de l'Angola et du Mozambique (rapatriés en majorité sans trop de problèmes en 1974-75), les gens pauvres des Açores ont fui vers l'Amérique, ceux de Madeira vers le Venezuela et l'Afrique du Sud. Après la seconde guerre mondiale, l'émigration découvre ses principales destinations en Europe : la France, surtout, mais aussi l'Allemagne, la Suisse, le Luxembourg, la Belgique. Sous le régime démocratique fondé par la révolution des œillets de 1974, cette tendance s'estompe : le pays se découvre même comme un lieu progressif de destination (ou de passage) des gens du Sud (africains, brésiliens, etc.). Mais ce n'était que partie remise. Comme il était de tradition chez les méditerranéens (italiens, grecs, etc.) et du centreorient de l'Europe continentale (allemands, polonais, slaves, juifs...), les portugais ont repris les chemins de l'étranger dès que la crise économique a frappé à nouveau, lors de ces toutes dernières années : des chômeurs de l'industrie et du bâtiment peu qualifiés, mais aussi des professionnels de moyenne-haute qualification qui ne trouvent plus dans le pays les salaires et les conditions matérielles de vie auxquelles ils considèrent avoir droit, après des années d'études éprouvées et le plus souvent l'effort financier des parents misant sur une scolarité prolongée de leurs enfants.

L'évocation du phénomène migratoire peut adroitement constituer un point de départ de l'ébauche d'analyse qui suit, sur les mouvements sociaux et les institutions de contrôle étatique des comportements de la population.

Et comment ? Oui, on sait bien que cela peut être théoriquement contesté mais l'on peut supposer que, dans les conditions du cas portugais évoqué (et tant d'autres semblables), l'émigration peut être interprétée en tant que mouvement social, ou peutêtre mieux, comme un proto-mouvement social. Cette idée prend appui sur les faits suivants :

16 1) la motivation essentielle des migrants est d'ordre économique, cherchant un pays différent du leur pour y travailler et vivre, ayant une attente sérieuse de rencontrer de meilleures conditions de travail et de rémunération, contenant implicitement un jugement critique envers le statu quo reçu et des actions pour le modifier.

17 2) le trajet et l'insertion dans le pays d'accueil n'est généralement pas un fait purement individuel, même si les décisions fondamentales le sont, puisqu'il s'agit de pratiques qui se déroulent la plupart du temps au travers de réseaux informels qui lient les points de départ et d'arrivée, qu'ils soient de nature familiale, de voisinage, de connaissances ou d'amitiés personnelles, totalement gratuites et solidaires, avec parfois quelques coûts à supporter. 
18 3) les communautés d'origine des partants continuent de constituer une base d'appui morale pour les émigrés, souvent aussi une référence symbolique durable ou encore un lieu d'ancrage avec un projet de retour en pleine intentionnalité, aussi bien sur le plan matériel qu'affectif ou de prestige, où la correspondance postale (et aujourd'hui le téléphone ou le Net) et les transferts de fonds d'épargne matérialisent physiquement les liens.

19 4) parallèlement, du fait des isolements ou des discriminations négatives subies dans le pays d'accueil, se constituent généralement des communautés d'immigrés, avec ou sans liaison au même point d'origine mais toujours dans le cadre d'une certaine identité nationale ou culturelle.

20 5) ces deux derniers points consolident l'idée d'enracinement du mouvement migratoire sur deux réalités sociales distinctes mais articulées entre elles par les agents-véhicules que sont les travailleurs migrants. Alors, avec de telles présuppositions, on peut dire que, tout comme les actions de grève ou les protestations collectives des salariés dans le cadre de la libre économie marchande, ces flux migratoires organisationnellement bipolarisés peuvent être considérés, eux aussi, comme des mouvements sociaux de l'ère moderne : avec l'aide de tiers, fuir les conditions de vie perçues comme mauvaises pour essayer d'en trouver de meilleures.

21 Nonobstant, il y a des cas semblables mais très particuliers qui doivent être détachés de ce modèle. Nous pensons aux travailleurs frontaliers, qui habitent d'un côté d'une frontière et travaillent régulièrement ailleurs, où il n'existe point de mouvement social mais seulement le fonctionnement d'un marché de travail particulier ; le cas des contrebandiers est identique, avec la différence que ceux-ci risquent leur peau.

Dans le Portugal moderne, comme on l'a dit, l'émigration est devenue structurelle, en partie légale mais en partie également illégale. Dans tous les cas, depuis 1886, une force veillait au contrôle policier des frontières, à côté des douanes : c'était la Garde Fiscale (militarisée et commandée par des officiers de l'armée) laquelle, en plus des tracasseries habituelles des petits porteurs de pouvoirs légaux, s'est toujours acharnée particulièrement sur les migrants illégaux, ceux qui quittaient le pays "a salto". Curieusement, une des mesures prises immédiatement par les capitaines qui ont fait la révolution démocratique du 25 Avril 1974 a été la dissolution de ce corps d'État. Justification politique : c'était le plus corrompu des instruments de répression du régime autoritaire créé par Salazar.

23 Le mouvement social du paysannat mérite que l'on s'y penche avec un peu plus d'attention. Disons d'abord qu'il n'y a jamais eu au Portugal un parti politique agraire, comme il est arrivé dans d'autres pays d'Europe. D'autre part, la puissance démographique de cette catégorie sociale a fait de lui, pendant longtemps, un agent politique indispensable pour tous ceux qui, dans les élites, se disputaient le pouvoir d'État. Sauf que cet agent n'a pratiquement jamais pu s'affirmer de manière autonome ; au contraire il s'est distingué par sa passivité, sa manière de s'abaisser aux curés et aux notables du village. Par comparaison, on peut penser aux descriptions que les grands écrivains russes du XIXe (Tolstoï, etc.) ont fait des moujiks et autres serfs, en rapport avec les seigneurs de la terre. Typiquement, la seule réelle manifestation d'autonomie des petits agriculteurs du centre-nord du pays (et aussi de l'Algarve) fût, vers 1850 la révolte armes-à-la-main dite de "Maria da Fonte» et les persistantes guérillas de «bons-brigands» comme José do Telhado ou João Brandão, politiquement conservateurs ou même réactionnaires. En général, les petits propriétaires-agriculteurs 
ont délégué la représentation de leurs intérêts aux mains des caciques locaux ou applaudissaient les paroles de Salazar vantant leurs habitudes d'humilité, de sacrifice et patriotisme et lui faisant confiance pour guider la nation, pourvu que l'on ne touche pas à leur petit lopin de terre. Tous les régimes politiques ont tenu cette promesse. Mais ces petits paysans ne se sont aperçus de leur destin de faillite qu'au moment où l'économie capitaliste les a contraints à migrer en ville ou à l'étranger, pour y chercher du travail comme salariés. Sachant encore que les travailleurs ruraux d'Alentejo ont rejoint adroitement les ouvriers d'usine quand la république est arrivée et que l'anarcho-syndicalisme battait son plein, il est facile de conclure que le mouvement social du paysannat n'a vraiment existé que sous forme potentielle et amputée de sa principale caractéristique : l'autonomie.

Malgré tout, où la persuasion n'était pas suffisante, le gouvernement faisait peser la raison d'Etat à travers des instruments de force existants. Pendant tout le XIX $\mathrm{X}^{\mathrm{e}}$ siècle, l'armée a été l'institution la plus utilisée pour le maintien de l'ordre, notamment dans les campagnes. Mais d'autres institutions surgirent, peut-être plus spécialisées dans cette besogne : jusqu'en 1839, une Garde Nationale a existé (semblable à celles alors existantes dans d'autres pays, connues comme "gardes bourgeoises»), mais elle a mal réussi, créant plus de désordres que le contraire. En substitution de celle-ci, une Garde Municipale est apparue en 1845, autant rurale qu'urbaine. Avec la république, naît la Garde Nationale Républicaine (GNR), également présente en milieu urbain et, peut-être surtout, en milieu rural : dans le premier cas, leur cavalerie était spécialement employée pour disperser des manifestations massives de protestataires ; dans les campagnes, c'était les patrouilles sillonnant les chemins ruraux qui marquaient d'avantage le paysage. C'est une force qui existe encore, toujours loyale au pouvoir politique en place et, avec la Police civile (1893), spécialisée dans le maintien de l'ordre en ville, elle est l'une des institutions les plus voyantes pour garantir la paix civile, la sécurité des citoyens et la bonne conscience des élites. Entre temps, une police criminelle existait depuis le XIX ${ }^{\text {e }}$, devenue Police Judiciaire dans le siècle suivant. Tout au contraire des travailleurs des champs, le mouvement ouvrier s'est affirmé comme un véritable mouvement social (conscience de soi, de l'adversaire et du champ historique qu'ils partageaient) à partir de la dernière décennie du XIXème et surtout après l'avènement de la république en 1910.

\section{XXe siècle}

L'organisation des syndicats, la grève, la solidarité, les manifestations de rue, les congrès, la presse et l'éducation figuraient dans la panoplie d'action des militants ouvriers ; mais quelques uns d'entre eux s'adonnaient aussi à des pratiques plus violentes, préparant «la révolution» ou bien répondaient à la répression gouvernementale ou patronale par le sabotage, la bombe ou des coups de révolvers.

Il est vrai que ce mouvement de résistance et de transformation sociale a voulu aussi avoir une présence et une représentation sur le terrain politique. Mais pendant longtemps, la nature de cette relation était plutôt ambivalente, ambiguë ou hésitante : le courant socialiste cherchait un certain équilibre entre le parti, les syndicats, les coopératives et les municipalités ; la tendance anarchiste affirmait nettement sa primauté sur les syndicats et ce n'est qu'avec les communistes-léninistes que la « courroie de transmission » vers les « fronts de masse » s'est imposée, dans les années 
1930. Cependant, cela n'a pas ôté au mouvement des travailleurs salariés (maintenant étendu aux employés du commerce, des bureaux, des banques et même les fonctionnaires d'État) les caractéristiques essentielles de mouvement social. Bien entendu, certaines catégories de ces travailleurs ont joué un rôle dans le mouvement social plus important que d'autres : au Portugal, cela a été le cas des typographes, des ouvriers du bâtiment, de ceux du liège, des "métallos", des cordonniers ou des cheminots. Même si cantonnés dans des communautés plus à l'écart, les mineurs, les pêcheurs, les dockers et les marins ont souvent été aussi à la pointe des luttes prolétariennes. Puisque la composition sociale du travail était en train de changer, dans les dernières décennies ce sont les professeurs, les infirmiers ou les fonctionnaires qui ont animé les revendications syndicales, au point où l'on est en droit de se demander si, dans ces nouvelles conditions, avec de nouveaux protagonistes et enjeux, il s'agit encore d'un mouvement social ou, plus simplement, d'action corporatistes dans le cadre d'un marché du travail de type nouveau.

Naturellement, comme très tôt Alain Touraine l'a signalé, quand le régime politique ne reconnaît pas son existence, ne respecte pas son autonomie ou essaye de l'intégrer dans les rouages de l'État - cas du corporatisme fascisant et des régimes socialistes ou autres de parti unique -, dans ces cas-là, la première revendication des travailleurs et de leurs leaders est celle de renverser cet état de choses, comme l'ont fait les militants de Solidarnosc en Pologne ou ceux de l'UGT dans l'Espagne franquiste. D'ailleurs, soit dit en passant, cela se produisait avec le soutien idéologique de la Déclaration Universelle des Droits Humains de l'ONU qui intègre le libre droit à constituer et d'adhérer (ou non) aux associations syndicales pour défendre les intérêts collectifs des salariés (approuvé en 1948 avec le vote de la délégation d'URSS, peut-être un peu distraite à ce momentlà). C'est pour ces raisons que les anarcho-syndicalistes de la CGT portugaise ont lutté par tous les moyens à leur portée contre la dictature jusqu'en 1948 ou 49, et que les communistes ont fait de même (y compris pratiquant « l'entrisme » dans les syndicats officiels corporatistes), tactique qui leur a valu d'apparaître parmi les vainqueurs au moment de la Révolution des đillets.

La capacité de résistance et de lutte de ce mouvement, même dans les conditions les plus difficiles (licenciements, prison, tortures, déportations, etc.), a naturellement déclenché de la part des gouvernements des réponses institutionnelles à la hauteur. Aux premiers temps de la république parlementaire, des milices de « révolutionnaires civils » ont persécuté les militants ouvriers et, avec la Grande Guerre, il vient une première police politique (sous couvert de services d'informations militaires). Dans les années 20 , plusieurs autres institutions du même type sont essayées, mais c'est la PVDE (Police de Vigilance et Défense de l'État) qui, à partir de 1932, devient de plus en plus puissante, active et féroce. Bien sûr, leurs cibles étaient tous ceux (républicains, socialistes, anarchistes et même quelques "fascistes purs») qui s'opposaient au gouvernement de la dictature mais, parmi eux, les travailleurs qui essayaient de résister ont été sans doute les plus atteints. Aux côtés de cette police (appelée plus tard PIDE - Police Internationale et de Défense de l'État - et encore DGS - Direction Générale de Sécurité), une nouvelle milice a pris place, la Légion Portugaise, guidée par une idéologie nationaliste et autoritaire, qui a fait aussi beaucoup de dégâts dans les rangs des personnes d'esprit libre ou démocratique, autant par la violence que par l'infiltration et la dénonciation. Mais dans les dernières années du salazarisme, la PIDE, en plus de ces missions déjà calées, a dû s'investir aussi dans les colonies pour contrer l'action des nationalistes africains, lesquels bien souvent ont été victimes de sévices 
cruels qui étaient épargnés aux blancs. Les "turras» (abrégé de terroristes, pour les guérilleros noirs) prenaient maintenant la place des antifascistes et des ouvriers récalcitrants. Mais il faut encore dire que la coordination entre ces différentes forces (et avec l'armée, pendant les guerres coloniales de 1961-1974) n'a pas toujours été efficace. Par exemple, après l'attentat contre Salazar, la rivalité entre la police politique et la police judiciaire a profité à l'anarchiste, et son auteur, a fini tout de même par être condamné à quinze années de prison, mais sans déportation outre-mer.

En régime démocratique, depuis 1974-76, ces empêchements ont disparus. Comme dans tous les pouvoirs d'État constitués, il y a des services "d'intelligence ", la Police Judiciaire et un nouveau Service d'Étrangers et des Frontières (SEF), dorénavant en charge des affaires de ce type (immigration, trafics, etc.), ainsi qu'une corporation des gardiens de prisons et une Police Maritime agrandie. La Police civile (PSP) et la GNR sont devenues des corps armés beaucoup plus développés, respectueux de l'ordre publique démocratique et, aujourd'hui, servis par un contingent non négligeable d'agents-femmes, qui ont aidé à une meilleure professionnalisation des accomplissements de ces institutions.

30 Prenons maintenant le cas de la jeunesse, et plus exactement celui du mouvement étudiant. Il ne s'agit pas ici d'une classe sociale, au sens sociologique le plus général, mais d'un groupe d'âge ou d'une catégorie sociale, situation transitoire pour chacun des membres qui, à un moment donné, le composent. Nonobstant, avec un tas de caractéristiques propres, ces mouvances ont souvent constitué un acteur social et politique important, que l'on peut aisément - pensons-nous - adjoindre aux restants des mouvements sociaux de la modernité.

31 Au Portugal, l'origine sociale des étudiants a été longtemps un quasi-monopole des classes moyennes (encore bien restreintes) et supérieures de la société. Malgré cela, au début du $\mathrm{XX}^{\mathrm{e}}$ siècle de forts mouvements font basculer l'institution universitaire et trembler des gouvernements. Le sens politique de ces actions était généralement progressiste, souvent républicain, anarchiste ou, à la fin, communiste. Mais, à partir des années 1920, un vent nationaliste souffle dans les milieux organisés de la jeunesse, et cela dure jusque dans les années 60 . Toujours très idéologisés, les jeunes de l'enseignement supérieur agitent alors très fortement l'ambiance urbaine de Lisboa, Coimbra et Porto, aiguillonnés par le refus de la guerre d'outre-mer et avec la composante non négligeable d'une nouvelle génération de catholiques (dits "progressistes »). L'extrême-gauche politique - surtout maoïste avec ses diverses tonalités - se développe alors considérablement, avec les communistes, quelques trotskistes, des adeptes de «l'action armée » et pratiquement pas d'anarchistes. En dépit de cela, dans « l'été chaud » de 1975 on parle beaucoup « d'anarcho-populisme »: en fait, c'était un peu l'esprit « Mai-68 » qui revenait, mais c'était des "groupuscules » qui tiraient les ficelles des luttes populaires dans les quartiers, les entreprises et les casernes, tandis que le parti communiste assurait les bases de son pouvoir social dans les syndicats, les unités de la réforme agraire, les municipalités et divers départements du secteur public. En tout état de cause, il est clair que les étudiants (et, de manière plus large, la jeunesse) ont été un acteur décisif et fondamental des mutations sociales dans le sens de la modernisation - que le Portugal a connu dans cette période, et incorporé ensuite.

Pourtant, au delà de la mobilisation politique (pour beaucoup assez passagère, disonsle), ce sont ces mêmes couches de jeunes déjà moyennement scolarisés qui ont porté en 
avant les drapeaux des nouveaux mouvements sociaux qui arrivaient aussi de l'étranger (par la télé, mais aussi par les canaux des migrations). Même si leurs combats ont été souvent perdus ou égarés, il faut noter - pour une fois - que les portugais ont alors rattrapé les retards historiques qui toujours les avaient désavantagés (rappelons, l'industrialisation, l'éducation ou la science) et ils ont suivi en peu de temps ce qui se passait aux États-Unis ou en Europe occidentale. Nous pouvons noter le cas du féminisme (droits des femmes, égalité, famille, lutte contre les discriminations, etc.), de l'écologisme (contre les pollutions, les risques de l'énergie nucléaire, les changements climatiques, la protection des espèces menacées, etc.) du pacifisme (pour le désarmement et la démilitarisation de la société), de l'émancipation sexuelle (contre les tabous) ou du solidarisme humanitaire (les droits humains, l'antiracisme, aide aux pays pauvres, etc.).

Dans le mouvement de libération des femmes ont compté les positions publiques assumées par quelques écrivains, relayées en démocratie par des personnalités de différents partis et de l'extrême-gauche, mais aucune formation politique n'a émergé sous ce label. Néanmoins, la législation a évolué rapidement dans le sens de l'égalité des droits, mais moins dans les pratiques sociales. Chez les "écolos", la mouvance était portée surtout par des groupes locaux autonomes souvent branchés sur des problèmes spécifiques (de pollution, contre le nucléaire, etc.). On discutait beaucoup sur l'éventuelle présence sur le terrain politique, mais un jour, de façon inopinée, est apparu un parti «Vert» (PEV) sorti de rien : c'était le parti-fraude créé en secret par le PC pour s'attirer quelques nouveaux sympathisants de ce côté, à qui il emprunte toute la logistique et qu'il accompagne fidèlement depuis lors aux élections et avec ses trois ou quatre députés au parlement (élus sur des listes communes). Mais le thème était tentant, touchait beaucoup de monde, le presse l'a promu et tous les partis traditionnels l'ont adopté dans leurs programmes. Quant au pacifisme, les mobilisations des années 70-80 ont été portées surtout par des gens d'extrême-gauche antiaméricainistes, mais il est vrai que les jeunes acceptaient de moins en moins le régime des casernes et le service militaire obligatoire a fini par être aboli. La libération sexuelle a aussi suivi son cours, avec des lobbies travaillant pour l'évolution de la législation et il y eu pas mal de résistances dans les comportements sociaux. Enfin, le solidarisme humanitaire a trouvé des causes et réussi beaucoup de bonnes réalisations, souvent portées par des catholiques mais aussi des laïques, comme ça a été le cas d'Amnesty International, de SOS-Racisme, d'Assistance Médicale Internationale (AMI) et de beaucoup d'autres organisations non-gouvernementales dédiées à l'aide des gens d'Afrique ou d'ailleurs en détresse. Les demandes d'aide financière ont toujours eu une réponse favorable du public (y compris dans le cas de l'indépendance du Timor-lest).

L'on peut toujours discuter si ces mouvements correspondent encore à une phase tardive de la modernité - disons, post-industrielle, pour employer des mots consacrés ou s'ils s'articulent déjà aux caractéristiques culturelles de ce qu'on appelle la postmodernité. Mais, en tous cas, leur pluralité et composition sociale (inter-classiste) contrastent avec l'homogénéité et le lieu central que le mouvement social des travailleurs salariés a occupé pendant de longues décennies dans les dynamiques et les conflits de nos sociétés. 


\section{Inventaire}

Nous touchons à la fin de cet inventaire socio-historique. Bien sûr, comme dans d'autres pays, les grandes institutions ont été toujours là pour régulariser les relations sociales et les références des humains : le gouvernement, le parlement, un chef d'État, la justice, les polices, les forces armées, l'Église et ses organisations d'aide sociale, les municipalités, l'université et le réseau scolaire, les hôpitaux, les musées et autres centres de culture, etc. Traversant quatre régimes politiques différents - monarchie constitutionnelle, république parlementaire, dictature et démocratie - ces institutions ont évolué et acquis des traits particuliers selon les époques mais chacune a gardé l'essentiel de sa mission spécifique. La population s'y est ajustée, tant bien que mal, en utilisatrice de leurs services, râlant de leurs insuffisances, en exigeant de nouveaux et de meilleurs services de qualité, applaudissant aux moments de joie et de gloire, et faisant le "gros dos » quand les tempêtes grondaient. Bien sûr, beaucoup d'initiatives et d'organisations volontaires de la société civile se sont toujours développées, mêmes si souvent elles ont cherché des parrainages officiels. Aussi les entrepreneurs ont porté le fardeau de la création d'un surplus économique, mais fréquemment on les a vu demander des protections ou des exemptions fiscales auprès des politiciens, en même temps qu'ils exerçaient une coercition sur leurs salariés. Les classes supérieures et moyennes ont toujours su défendre leurs intérêts, y compris lorsqu'elles se battaient entre elles. Après l'abaissement de l'aristocratie (au long du XIX ${ }^{\mathrm{e}}$ siècle) l'espace de la représentation politique (les partis, les élections, les élus) a été progressivement monopolisé par une "classe politique » qui a tiré profit de sa mainmise sur les rouages de l'État et des services publics.

C'est à l'encontre de ces tendances, ici grossièrement évoquées, que des fractions un peu plus éclairées du peuple et de l'intelligentsia ont essayé de réagir, en déclenchant des efforts de propagande, d'action et d'organisation autonomes qui constituèrent finalement les mouvements sociaux de la modernité. Sans elles, l'histoire des deux derniers siècles aurait été bien différente. Aucune "tâche historique " ne leur était assignée. Parfois, ces mouvements sociaux se sont égarés, trompé d'adversaire, ils ont rendu leurs forces dans les mains de quelque "sauveur suprême » et se sont souvent divisés dans leurs organisations intérieures parce que "l'unité d'action" était toujours un palier qu'il fallait gravir, avec des efforts et le sacrifice de quelques uns. Mais ils ont apporté une dynamique et un désir de changement à la vie sociale comparables seulement à ceux que les forces libérées de l'économie avaient démontrés au moins depuis la " révolution industrielle».

Le Portugal est un cas d'espèce, singulier, mais il reproduit à sa manière ce qui s'est passé généralement dans l'Occident moderne. Un chemin qui n'a pas encore fini de nous surprendre.

DUFOULON, Serge (1998), Les gars de la marine: ethnographie d'un navire de combat, Paris, Métailié.

FREIRE, João (2002), Les anarchistes du Portugal, Paris, CNT-RP.

FREIRE, João (2003), Homens em Fundo Azul Marinho (Des hommes sur fond bleu marine), Oeiras Celta. (essai d'observation interne au millieu du XXême siècle)

FREIRE, João (2007), Pessoa Comum no seu Tempo, Porto, Afrontamento. (mémoires d'un moyenbourgeois de Lisbonne dans la seconde moitié du XXème) 
FREIRE, João (2009), « Militares e intervenção política », Revista Crítica de Ciências Sociais, Coimbra, $\mathrm{n}^{\mathrm{o}}$ 86: 3-23. (bilan d'un siècle d'interventionnisme politique des militaires au Portugal)

FREIRE, João (2010), A Marinha e o Poder Político em Portugal no Século XX, Lisboa, Colibri. (analyse du rôle politique de la marine)

FREIRE, João (2013), Do Controlo do Mar ao Controlo da Terra, Lisboa, Edições Culturais da Marinha. (entre la chasse au trafic d'esclaves et l'imposition de souveraineté dans le nord du Mozambique, 1840-1930)

FREIRE, João (2016), Jornal da Marinha, Lisboa, Colibri. (analyse institutionnelle de ce corps militaire, 1834-2014)

FREIRE, João (2017), A Colonização Portuguesa da Guiné 1880-1960, Lisboa, Edições Culturais da Marinha.

FREIRE, João \& LOUSADA, Maria Alexandre (2013), Roteiros da Memória Urbana; Vol. I, Lisboa; Vol. 2, Porto; Vol. 3, Setúbal, Lisboa, Colibri. (trois guides pour retrouver des jalons libertaires dans les tissus urbains)

\section{NOTES}

1. FREIRE, João (2003), Homens em Fundo Azul Marinho (Des hommes sur fond bleu marine), Oeiras Celta. (essai d'observation interne au millieu du XXême siècle)

\section{INDEX}

Mots-clés : mouvements sociaux, action collective, histoire du combat social, Portugal moderne, militaires

\section{AUTEUR}

\section{JOÃO FREIRE}

Sociologue, Professeur émérite à ISCTE - Institut Universitaire de Lisbonne 